\title{
Erratum to: Auctioning Risky Conservation Contracts
}

\author{
Bruno Wichmann ${ }^{1}$ - Peter Boxall ${ }^{1}$ - Scott Wilson ${ }^{1}$. \\ Orsolya Perger ${ }^{1}$
}

\section{Erratum to: Environ Resource Econ DOI 10.1007/s10640-016-0063-x}

The 4th author name is Orsolya Perger and not Orsolya Pergery as typeset in the original paper.

The online version of the original article can be found under https://doi.org/10.1007/s10640-016-0063-x.

$凶 \quad$ Bruno Wichmann

bwichmann@ualberta.ca

Peter Boxall

pboxall@ualberta.ca

Scott Wilson

scott.wilson@ualberta.ca

Orsolya Perger

operger@ualberta.ca

1 Department of Resource Economics and Environmental Sociology, University of Alberta, 503 General Services Building, Edmonton, AB T6G 2H1, Canada 